[Article]

www.whxb.pku.edu.cn

\title{
ZnO 自组装薄膜的可控生长及其光催化性能
}

\author{
谢娟 ${ }^{1,2}$ 王 虎 ${ }^{1,2, *}$ 段 明 ${ }^{1}$
}

('西南石油大学, 油气藏地质及开发工程国家重点实验室, 成都 610500; “西南石油大学材料科学与工程学院, 成都 610500)

\begin{abstract}
摘要：通过改变工艺参数, 制得了粒径可控的 ZnO 自组装薄膜. 该薄膜在可见光区域出现了光子带隙. 以染 料甲基橙的光催化降解为模型评价了 $\mathrm{ZnO}$ 自组装薄膜的光催化活性. 利用 $\mathrm{X}$ 射线衍射仪 (XRD)和扫描电子显 微镜(SEM)表征了 $\mathrm{ZnO}$ 的晶体结构和微观形貌. 实验结果表明, $\mathrm{ZnO}$ 自组装薄膜在太阳光照射下表现出良好 的光催化性能, 其光催化活性随着 $\mathrm{ZnO}$ 颗粒粒径的减小而提高. $\mathrm{ZnO}$ 自组装薄膜光催化降解甲基橙的反应符 合一级反应动力学规律.
\end{abstract}

关键词: ZnO; 自组装; 可控生长; 光子带隙; 光催化 中图分类号: 0643

\section{Controlled Growth of Self-Assembled ZnO Thin Films and Characterization of Their Photocatalytic Properties}

\author{
XIE Juan ${ }^{1,2} \quad$ WANG Hu H, $^{1, *} \quad$ DUAN Ming ${ }^{1}$ \\ ('State Key Laboratory of Oil and Gas Reservoir Geology and Exploitation, Southwest Petroleum University, \\ Chengdu 610500, P. R. China; ${ }^{2}$ College of Materials Science and Engineering, Southwest Petroleum University, \\ Chengdu 610500, P. R. China)
}

\begin{abstract}
Self-assembled $\mathrm{ZnO}$ thin films with controlled sizes were successfully prepared by varying the processing parameters. The films have a photonic band gap, which extends the absorption range to the visible light region. The photocatalytic activities of the $\mathrm{ZnO}$ thin films were evaluated by the degradation of methyl orange (MO). The crystal structure of $\mathrm{ZnO}$ was characterized by X-ray diffraction (XRD) and scanning electron microscopy (SEM). The results show that the $\mathrm{ZnO}$ thin films exhibit good photocatalytic activities under sunlight. Furthermore, the photocatalytic activities of the $\mathrm{ZnO}$ thin films were highly dependent on sphere size. With an increase in $\mathrm{ZnO}$ sphere size, the degradation efficiency toward MO decreased. The photodegradation can be described using a pseudo-first-order kinetics equation.
\end{abstract}

Key Words: ZnO; Self-assembly; Controlled growth; Photonic band gap; Photocatalysis

随着社会和经济的发展, 环境污染日益严重, 不仅危害人类的身体健康, 而且阻碍了经济的发 展. 高色度印染废水对环境污染危害严重, 但因其 成分复杂、色泽深, 给工业废水的常规处理带来了 很大的难度 ${ }^{[1-3]}$. 目前, 我国对印染废水的常规处理 方法主要有氧化法、反渗透法、吸附沉淀法和中和
法等 ${ }^{[4-6]}$. 水溶性偶氮染料是印染废水污染治理的主 要对象, 由于其含有难以降解的苯环, 导致使用化 学和生物等降解方法难以达到理想的效果, 寻求新 的水处理方法成为十分迫切的问题 ${ }^{[7-10]}$.

在 20 世纪 80 年代后期, 光催化氧化法开始应 用于控制环境污染领域. 不同于传统的处理方法,

Received: July 27, 2010; Revised: October 18, 2010; Published on Web: December 8, 2010.

"Corresponding author. Email: senty78@126.com; Tel: +86-28-66896950.

The project was supported by the Open Fund of State Key Laboratory of Oil and Gas Reservoir Geology and Exploitation (Southwest Petroleum University, SWPU), China (PLN0805).

油气藏地质及开发工程国家重点实验室开放基金(西南石油大学)(PLN0805)资助项目

(C) Editorial office of Acta Physico-Chimica Sinica 
光催化氧化技术能有效地破坏许多结构稳定的难 降解污染物, 具有降解效率高和污染物降解彻底等 优点, 引起了广泛的关注 ${ }^{[1-14]}$. 光催化氧化技术已在 降解有机污染物等领域取得了良好的效果. 当光催 化剂被适当的光源照射后, 能产生光生电子-空穴 对. 光生空穴作为一类强氧化剂能和水反应生 成. $\mathrm{OH}$ 自由基, 通过一系列氧化过程最终将染料等 有机物降解 ${ }^{[15]}$.

近年来, 已发现多种光催化剂, 如 $\mathrm{TiO}_{2} 、 \mathrm{ZnO}$ 、 $\mathrm{CdS} 、 \mathrm{WO}_{3}$ 等. 由于多数光催化剂的禁带宽度位于紫 外区域, 只能吸收紫外光. 然而, 太阳光中紫外光只 有 4\%. 因此, 光催化剂只能吸收紫外光的本征特性 在很大程度上限制了它们的应用. 能否提高光催化 剂对太阳光(尤其是可见光)的利用率直接影响了光 催化剂的光催化效率, 这也是目前光催化氧化领域 的研究热点 ${ }^{[16-21]}$.

$\mathrm{ZnO}$ 作为一种重要的半导体材料, 由于其优异 的性能已被广泛地应用于压电传感器、光电器件和 催化剂等领域. $\mathrm{ZnO}$ 具有低成本和在酸碱性介质中 均表现出良好的催化降解性能, 被认为是极具应用 前景的高活性光催化剂. 遗憾的是, $\mathrm{ZnO}$ 的禁带宽 度为 $3.2 \mathrm{eV}$, 故 $\mathrm{ZnO}$ 只能吸收紫外光. 因而, 众多学 者致力于提高 $\mathrm{ZnO}$ 在可见光区域的光催化性能的 研究 ${ }^{[22]}$.

本文利用胶体自组装法得到了粒径可控的 $\mathrm{ZnO}$ 薄膜. 所得的 $\mathrm{ZnO}$ 自组装薄膜具有可调控的可 见光波段的光子带隙, 为实现 $\mathrm{ZnO}$ 在可见光区域的 光催化降解污染物提供了条件. 甲基橙是一种典型 的水溶性偶氮染料, 其色度深、不易在自然条件下 用微生物去除或分解. 本文以光催化降解甲基橙溶 液为模型, 探讨了 $\mathrm{ZnO}$ 自组装薄膜在不同的光源照 射下的光催化降解性能, 以期为发展新型环境纳米 材料提供理论依据.

\section{1 实 验}

\section{$1.1 \mathrm{ZnO}$ 自组装薄膜的可控生长}

$\mathrm{ZnO}$ 自组装薄膜的可控生长主要包括三个步 骤 ${ }^{[23-24]}$. (1) 清液的制备, 将醋酸锌(分析纯)和一缩二 乙二醇(DEG, 分析纯)按 $1: 30$ (质量比)混合后缓慢加 热至 $180^{\circ} \mathrm{C}$, 这时混合物呈乳白色溶胶状. 将该溶胶 体系离心分离后, 留取上层清液 (溶液 $\mathrm{A}$ ) 作为后续 反应的反应物. (2) $\mathrm{ZnO}$ 胶体球的可控生长, 按相同 比例称取醋酸锌和 DEG, 两者混合后缓慢加热至 $170^{\circ} \mathrm{C}$, 取适量的溶液 $\mathrm{A}$ 加热到混合物中, 再将温度 升至 $180^{\circ} \mathrm{C}$, 保温 $1 \mathrm{~h}$, 得到乳白色的含有 $\mathrm{ZnO}$ 胶体 球的悬浮溶液. 可以通过改变溶液 $\mathrm{A}$ 的加入量 (1-20 mL) 来调节 $\mathrm{ZnO}$ 胶体球的粒径大小, 从而实 现可控生长. (3) $\mathrm{ZnO}$ 薄膜的自组装生长, 利用恒温 加热平台维持基底温度为 $60^{\circ} \mathrm{C}$, 将含有 $\mathrm{ZnO}$ 胶体 球的悬浮溶液滴加到石英玻璃基底表面. 待溶剂挥 发完全后, 将基底温度升至 $150{ }^{\circ} \mathrm{C}$ 进行热处理, 去 除表面吸附的残余有机溶剂. 最终, 在石英基底表 面自组装生长出 $\mathrm{ZnO}$ 薄膜.

\section{2 光催化性能的测试}

以染料甲基橙的光催化降解为模型评价 $\mathrm{ZnO}$ 的光催化活性. 光催化性能测试在不透光的反应箱 中进行. 以 $125 \mathrm{~W}$ 高压录灯作为光源(主波长为 365 $\mathrm{nm}$ ), 为增大样品的光照面积用细铜丝悬吊薄膜样 品, 使之与录灯平行, 薄膜处于液面以下 $1.5 \mathrm{~cm}$ 处 且与录灯相距约 $15 \mathrm{~cm}$. 反应过程中持续磁力摚拌, 定时取样, 通过甲基橙的浓度随反应时间的变化来 研究 $\mathrm{ZnO}$ 光催化性能. 甲基橙的浓度随反应时间的 变化采用直接比色法在紫外-可见分光光度计上跟 踪分析. 根据甲基橙水溶液的标准曲线, 可得到不 同反应时间下甲基橙的浓度, 再由下式计算甲基橙 的降解率 $(\eta)$ :

$$
\eta=\frac{C_{0}-C_{t}}{C_{0}} \times 100 \%
$$

式中, $C_{0}$ 和 $C_{t}$ 分别为甲基橙溶液在 $465 \mathrm{~nm}$ 处的初始 浓度和降解 $t$ 时刻后的浓度.

\section{3 结构与性能的表征}

利用 $\mathrm{X}$ 射线衍射仪 $\left(\mathrm{XRD}\right.$, 辐射源为 $\mathrm{Cu} K_{\alpha}$, $\mathrm{XRD}-7000 \mathrm{~S} / \mathrm{L}$, 日本岛津公司)测定 $\mathrm{ZnO}$ 自组装薄膜 的晶体结构, 扫描电子显微镜(SEM, JSM-6490LV, 日本电子株式会社 JEOL) 观测 $\mathrm{ZnO}$ 自组装薄膜的微 观形貌. 利用紫外可见分光光度计(UV2550, 日本岛 津公司)测定 $\mathrm{ZnO}$ 薄膜的紫外-可见透射光谱.

\section{2 结果与分析}

\section{$2.1 \mathrm{ZnO}$ 自组装薄膜的表征}

图 1 是溶液 $\mathrm{A}$ 用量为 $15 \mathrm{~mL}$ 时所得的 $\mathrm{ZnO}$ 自组 装薄膜的 X 射线衍射图谱(XRD). 从 XRD 图谱上可 知, 各个衍射峰的位置和强度与标准的六角相纤锌 矿 ZnO(JCPDS No.5-0664)的特征峰一一对应, 表明 生成的 $\mathrm{ZnO}$ 为六方晶体结构. 根据以下德拜-谢乐公 式 ${ }^{[25]}$ 可以计算出晶粒的尺寸. 


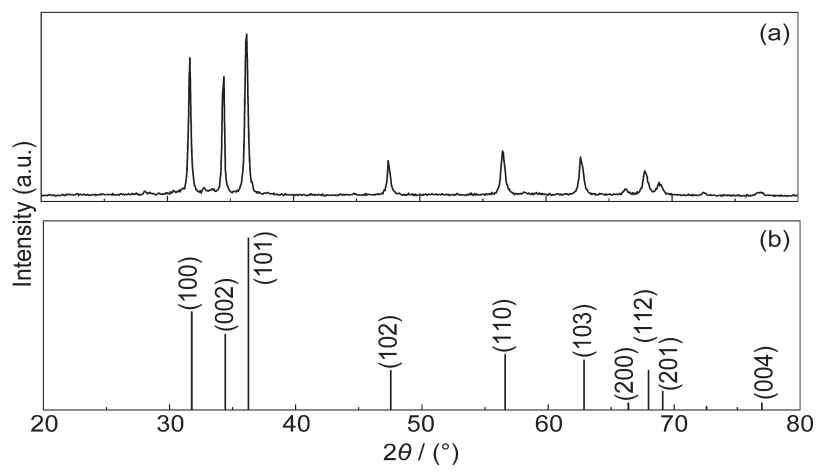

图 1 (a) 自组装法制备的 $\mathrm{ZnO}$ 样品的 XRD 图谱和 (b) ZnO 的标准 XRD 图谱(JCPDS No.5-0664)

Fig.1 XRD pattern of (a) $\mathrm{ZnO}$ crystals prepared by self-assembly method and (b) standard pattern of powder ZnO (JCPDS No.5-0664)

$$
D=\frac{0.9 \lambda}{B \cos \theta_{\mathrm{B}}}
$$

其中, $D$ 为晶粒的直径, $\lambda$ 为 $\mathrm{X}$ 射线衍射波长 $(0.154178 \mathrm{~nm}), B$ 为衍射峰的半高宽, $\theta$ 为布拉格衍 射角. 由图上 $\mathrm{ZnO}$ 的(101)衍射峰的半高宽计算出平 均晶粒直径为 $29 \mathrm{~nm}$.

当溶液 $\mathrm{A}$ 用量为 $15 \mathrm{~mL}$ 时, 石英基底上生长的 $\mathrm{ZnO}$ 自组装薄膜的扫描电镜图(SEM)如图 2 所示. 自 组装法生长的 $\mathrm{ZnO}$ 呈粒径均匀的球状 (直径约为 $400 \mathrm{~nm}$ ), 且有序紧密排列. 高倍数的 SEM 可以更好
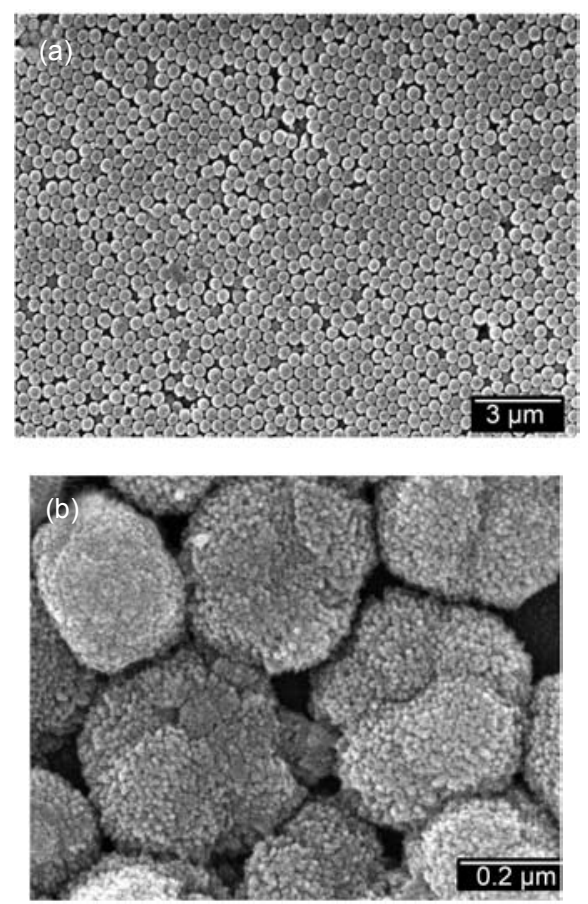

图 2 石英玻璃表面自组装法制备的 ZnO 样品的 SEM 图

Fig.2 SEM images of $\mathrm{ZnO}$ crystals prepared on quartz glass by self-assembly method

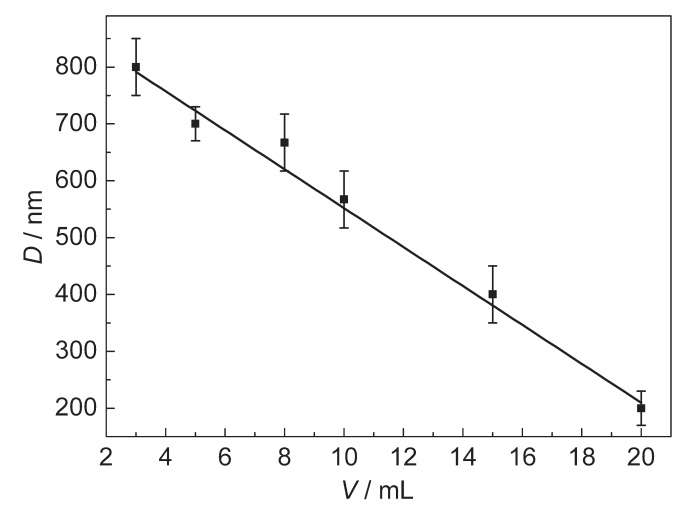

图 $3 \mathrm{ZnO}$ 二次粒子的尺寸 $(D)$ 与溶液 $\mathrm{A}$ 用量的关系曲线

Fig.3 Relationship between sizes of $\mathrm{ZnO}$ secondary particles $(D)$ and volume of the solution $A$

Solution A consisted of crystal nucleus and polydisperse $\mathrm{ZnO}$ singlets.

地表征微观形貌(如图 2(b)所示). 每个 $\mathrm{ZnO}$ 胶体球 都是由大量纳米级的小晶粒构成的二次粒子. 这些 小晶粒的粒径约为 $30 \mathrm{~nm}$, 与 XRD 的结果相符.

在其它条件不变的情况下, 改变溶液 $\mathrm{A}$ 的用 量, 可以制得不同粒径的 $\mathrm{ZnO}$ 胶体球. 图 3 为 $\mathrm{ZnO}$ 二 次粒子的平均粒径与溶液 $\mathrm{A}$ 用量的关系曲线. 由图 可知, 随着溶液 $\mathrm{A}$ 用量的减少, $\mathrm{ZnO}$ 胶体球的粒径逐 渐增大, 其粒径变化范围为 200-800 nm. 不难看出, 溶液 $\mathrm{A}$ 的用量对最终生成的 $\mathrm{ZnO}$ 二次粒子的尺寸 有较大的影响.

整个自组装法制备 $\mathrm{ZnO}$ 的过程包含了两个重 要的过程: (1) 初级微粒的形成阶段, 即多分散的 $\mathrm{ZnO}$ 胶体的形成. 随着化学反应的进行, 溶液中溶 质增多并达到饱和状态, 导致成核发生. 成核过程 发生得十分迅速, 并逐渐生长为初级微粒; (2) 次级 微粒的形成阶段, 即 $\mathrm{ZnO}$ 二次粒子的形成. 通过初 级多分散微粒自发地尺寸和形貌选择性聚集, 形成 了粒径均匀、排列整齐的 $\mathrm{ZnO}$ 二次粒子 ${ }^{[26]}$.

本文推测, 溶液 $\mathrm{A}$ 主要是由晶核和多分散的 $\mathrm{ZnO}$ 初级微粒构成的. 随着溶液 $\mathrm{A}$ 用量的减小, 即溶 液中所含的初级微粒的数目和成核的数目减少, 反 应基团生成速率加快, 初级微粒粒径增大, 最终导 致二次胶体粒子的粒径增大. 为了证实以上推测, 本文对不同粒径的二次粒子及相对应的初次微粒 的尺寸进行了统计, 得到了初次微粒粒径与溶液 A 用量的关系曲线, 如图 4所示. 由图可知, 随着溶液 $\mathrm{A}$ 用量的减小, 初次微粒粒径逐渐增大, 这与推测相 符.

\section{$2.2 \mathrm{ZnO}$ 自组装薄膜的光学带隙结构}




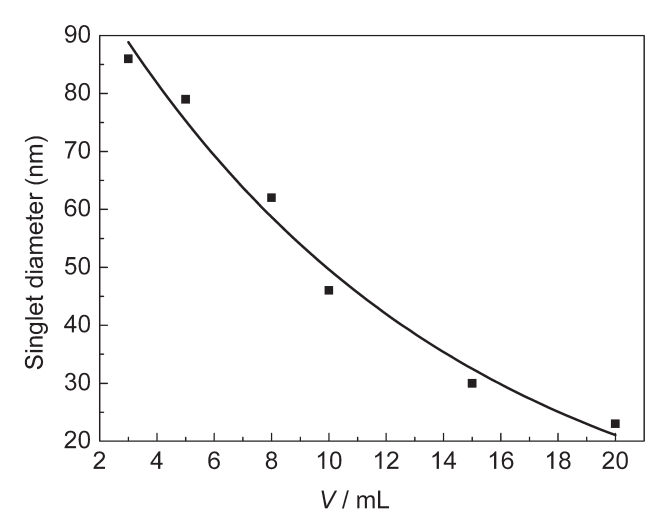

图 4 初级微粒粒径与溶液 $\mathrm{A}$ 用量的关系曲线

Fig.4 Relationship between diameter of $\mathrm{ZnO}$ singlets and volume of the solution $A$

在石英基底上以粒径为 $300 \mathrm{~nm}$ 的单分散溶胶 自组装生长薄膜, 对样品进行紫外-可见透射光谱测 试, 如图 5 所示. 从图中可以知道, 该样品的透射光 谱在紫外区域有一个吸收带边, 根据 $(\alpha h v)^{2}$ 与光子能 量的关系可以得到吸收带边的位置为 $375 \mathrm{~nm}$. 除了 上述的吸收带边外, 透射光谱在波长 $465 \mathrm{~nm}$ 附近还 有一个较强的吸收, 这对应于光子晶体的光子带 隙.

对不同粒径的 $\mathrm{ZnO}$ 自组装薄膜进行紫外-可见 透射光谱测试发现, $\mathrm{ZnO}$ 胶体球粒径不同, 其光子 带隙中心波长 $\lambda_{\mathrm{c}}$ 的位置也会发生变化. 图 6 为 $\mathrm{ZnO}$ 胶体球粒径 $D$ 与光子带隙中心波长 $\lambda_{\mathrm{c}}$ 的关系曲线. 由图可知, 随着 $\mathrm{ZnO}$ 胶体球粒径的增大, $\lambda_{\mathrm{c}}$ 的位置由 $465 \mathrm{~nm}$ 增大至 $1000 \mathrm{~nm}$, 这个范围几乎覆盖了整个 可见光区域, 并延伸到近红外波段. 中心波长 $\lambda_{\mathrm{c}}$ 的位 置随 $\mathrm{ZnO}$ 胶体球粒径的减小而发生蓝移现象(即 $\lambda_{\mathrm{c}}$ 的位置向较短的波长方向移动), 且该移动完全受胶 体球粒径的控制. 这是光子晶体本征特性的体现.

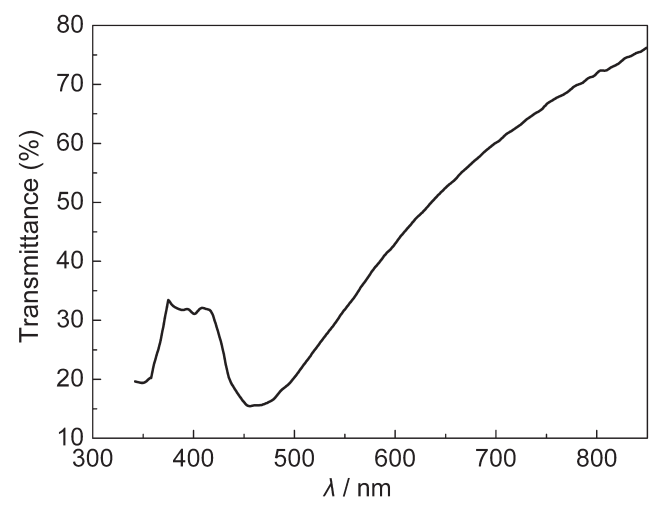

图 5 粒径为 $300 \mathrm{~nm}$ 的 $\mathrm{ZnO}$ 自组装薄膜的透射光谱 Fig.5 Transmission spectra for $\mathrm{ZnO}$ self-assembled film with the diameter of $300 \mathrm{~nm}$

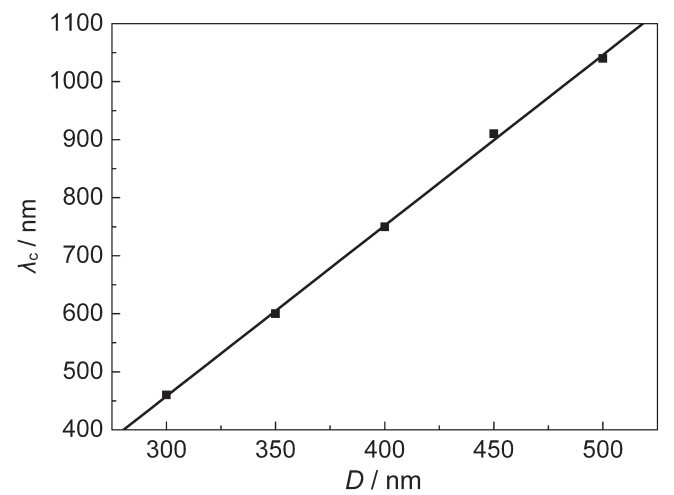

图 $6 \mathrm{ZnO}$ 胶体球直径 $D$ 与光子带隙中心波长 $\lambda_{c}$ 的关系曲线

Fig.6 Relationship between the photonic band position wavelength $\left(\lambda_{c}\right)$ and the diameter $(D)$ of $\mathrm{ZnO}$ colloidal spheres

由图 6 不难看出, $D$ 与 $\lambda_{\mathrm{c}}$ 呈线性关系. 由此可知, 通过 控制溶液 $\mathrm{A}$ 的用量, 最终实现了对光子带隙中心波 长 $\lambda_{0}$ 位置的调控.

\section{$2.3 \mathrm{ZnO}$ 自组装薄膜的光催化性能}

以光催化降解 $200 \mathrm{~mL}, 15 \mathrm{mg} \cdot \mathrm{L}^{-1}$ 的甲基橙溶 液来评价不同粒径 $(300 、 350 、 400 、 450 、 500 \mathrm{~nm})$ 的 $\mathrm{ZnO}$ 自组装薄膜的光催化性能. 图 7 为不同粒径的 $\mathrm{ZnO}$ 自组装薄膜分别在暗室中、太阳光和高压录灯 下照射 $60 \mathrm{~min}$ 后降解甲基橙的效率. 由图可知, 在 暗态下, 无论是否存在 $\mathrm{ZnO}$ 光催化剂, 甲基橙溶液 的浓度几乎没有变化, 其降解率均接近于 0 . 这说明 暗态下甲基橙溶液不会发生降解反应.

在太阳光照射下, 未加入光催化剂时, 太阳光 的照射并不能引起甲基橙溶液浓度的下降. 相反 地, 随着照射时间的延长, 甲基橙溶液的浓度略有 增加. 这主要是由于在光照下有少量溶剂蒸发, 从 而引起了甲基橙溶液浓度的升高. 当以 $\mathrm{ZnO}$ 自组装 薄膜作为光催化剂时, 甲基橙溶液的浓度减小, 发

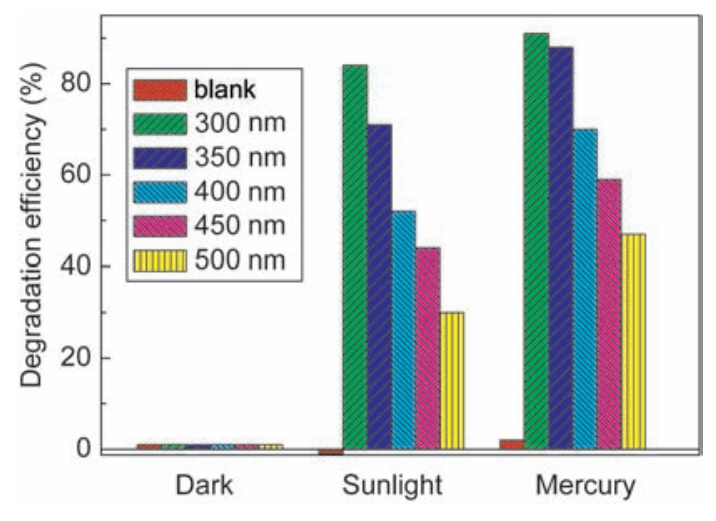

图 7 不同光源照射下甲基橙的光催化降解率

Fig.7 Degradation efficiency of methyl orange under different light sources 
生降解反应, 且随着 $\mathrm{ZnO}$ 粒径的增大, 甲基橙的光 催化降解率减小. 太阳光中大部分为可见光(波长为 400-700 nm), 紫外光所占比例很小. 能利用太阳光 降解甲基橙, 这可能与低维 $\mathrm{ZnO}$ 自组装薄膜对光子 带隙的调节作用有关. 可以推断出, 正是由于其对 可见光的吸收作用, 使得 $\mathrm{ZnO}$ 自组装薄膜在太阳光 照射下表现出光催化的性能.

在高压录灯照射下, 无 $\mathrm{ZnO}$ 光催化剂作用时, 甲基橙溶液浓度变化很小, 未发生明显的光解或光 氧化现象. 而甲基橙溶液在 $\mathrm{ZnO}$ 自组装薄膜的作用 下, 溶液出现了明显的光催化降解现象.

不同粒径的 $\mathrm{ZnO}$ 自组装薄膜的光催化降解率 具有较大差异. 在光催化降解 $60 \mathrm{~min}$ 后, 随着 $\mathrm{ZnO}$ 粒径的增大, 甲基橙溶液的降解率逐渐降低. $\mathrm{ZnO}$ 二次粒子粒径越小, 其纳米级的初次粒子的粒径也 越小. 甲基橙降解率随 $\mathrm{ZnO}$ 粒径的减小而增大的现 象可能与以下两方面原因有关: 一方面, 颗粒粒径 尺寸越小, 光生载流子从主体到达表面的时间越 短, 甚至小于光生载流子的复合时间(约为 $10 \mathrm{~ns}$ ), 这 样使得更多的光生空穴在半导体表面被捕获, 从而 发生氧化反应, 使其光催化活性更高; 另一方面, 颗 粒粒径尺寸越小, 其禁带宽度越大, 价带电位变得 更正, 空穴的氧化能力越强, 故其在高压永灯的照 射下的光催化活性越强.

为了研究 $\mathrm{ZnO}$ 自组装薄膜光催化降解甲基橙 的反应动力学规律, 以不同粒径的 $\mathrm{ZnO}$ 自组装薄膜 作为光催化剂, 在太阳光照射下对 $200 \mathrm{~mL}, 15 \mathrm{mg}$. $\mathrm{L}^{-1}$ 的甲基橙溶液进行降解测试. 实验所得数据经过 计算后结果如图 8 所示. 由图可知, $\ln \left(C_{0} / C_{t}\right)$ 与降解 反应时间 $t$ 之间有较好的线性关系, 而且相关系数 $R^{2}$ 的值都接近于 1 , 表明 $\mathrm{ZnO}$ 自组装薄膜光催化降 解甲基橙的反应符合一级反应动力学规律.

根据 Langmuir-Hinshelwood (L-H)动力学模型 假设, 反应速率可以表示为:

$$
r=k \theta=-\frac{d C}{d t}=\frac{k K C}{1+K C}
$$

其中, $\theta$ 为反应物在催化剂表面的吸附份额或称表 面覆盖度; $K$ 为吸附常数, 相当于吸附速率常数与脱 附速率常数的比值; $C$ 为溶液中反应物的浓度; $k$ 为 催化剂表面上活性位的表观反应速率常数, 包含诸 如催化剂用量、有效光子通量等参数.

当反应物溶液的初始浓度较低时, $K C<<1$, 故 式(3)可以写成:

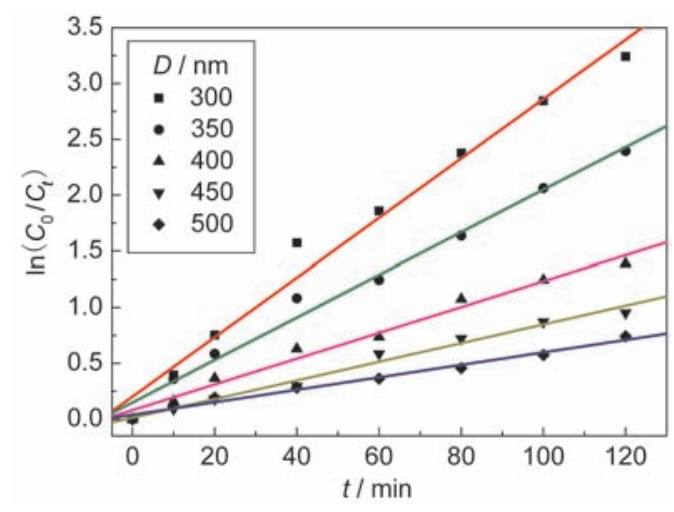

图 8 不同粒径的 $\mathrm{ZnO}$ 自组装薄膜降解甲基橙的 $\ln \left(C_{0} / C_{t}\right)$ 与 降解时间的关系曲线

Fig.8 Relationship between $\ln \left(C_{0} / C_{t}\right)$ and degradation time of methyl orange with different particle diameters of $\mathrm{ZnO}$ self-assembled thin films

$$
r=-\frac{\mathrm{d} C}{d t}=k K C=k_{\mathrm{a}}^{\prime} C
$$

其中, $k_{\mathrm{a}}^{\prime}$ 为假一级反应动力学常数.

对式(4)积分并整型为 $C=f(t)$ 的速率方程为:

$\ln \frac{C_{0}}{C_{t}}=k_{a} t$

式中, $k_{\mathrm{a}}$ 为反应速率常数 ${ }^{[27]}$.

由式(5)可知, $\ln \left(C_{0} / C_{t}\right)$ 与反应时间 $t$ 呈线性关 系, 且直线的斜率为一级反应的速率常数. 这与本 文实验数据相符, 即实验所得数据符合 L-H 模型的 相关理论.

由图 8 , 根据实验数据可拟合求出不同粒径 $\mathrm{ZnO}$ 自组装薄膜降解甲基橙溶液的动力学方程、速 率常数 $(k)$ 以及半衰期等参数, 如表 1 所示 $\left(\right.$ 半衰期 $t_{1 / 2}$ 是指反应物被降解为初始浓度的 $1 / 2$ 时所需时间, 即当 $C_{t}=C_{0} / 2$ 时, 对应的反应时间). 从表 1 中不难看 出, 随着 $\mathrm{ZnO}$ 粒径的减小, 反应速率常数逐渐增大, 催化反应速率逐渐加快. 根据 L-H 理论可知, L-H 模 型的基本前提条件是反应物在催化剂表面吸附符

\section{表 1 不同粒径的 $\mathrm{ZnO}$ 自组装薄膜对甲基橙降解的 反应速率常数}

Table 1 Rate constant values for the degradation of methyl orange with different particle diameters of ZnO self-assembled thin films

\begin{tabular}{ccccc}
\hline$D / \mathrm{nm}$ & \multicolumn{1}{c}{ Kinetic equation } & $k / \mathrm{min}^{-1}$ & $R^{2}$ & $t_{1 / 2} / \mathrm{min}$ \\
\hline 300 & $\ln \left(C_{0} / C_{t}\right)=0.0266 t+0.20127$ & 0.027 & 0.991 & 18.5 \\
350 & $\ln \left(C_{0} / C_{t}\right)=0.019 t+0.14922$ & 0.019 & 0.994 & 28.6 \\
400 & $\ln \left(C_{0} / C_{t}\right)=0.01153 t+0.08012$ & 0.012 & 0.991 & 53.2 \\
450 & $\ln \left(C_{0} / C_{t}\right)=0.00836 t+0.0119$ & 0.008 & 0.993 & 81.5 \\
500 & $\ln \left(C_{0} / C_{t}\right)=0.00558 t+0.03925$ & 0.006 & 0.993 & 117.2 \\
\hline
\end{tabular}


合 Langmuir 吸附等温线, 反应速率取决于吸附量的 大小. 而化合物在半导体表面上的吸附一般遵循 Langmuir 吸附等温式. 因此, $\mathrm{ZnO}$ 自组装薄膜对甲 基橙的光催化降解反应速率取决于甲基橙在 $\mathrm{ZnO}$ 表面的吸附量的大小. 由于粒径较小的 $\mathrm{ZnO}$ 颗粒的 比表面积相对较大, 故甲基橙在其表面的吸附量增 大, 因此, 光催化降解反应速率就会增大.

\section{3 结 论}

通过改变反应参数来调节 $\mathrm{ZnO}$ 光子带隙中心 波长的位置, 最终实现了 $\mathrm{ZnO}$ 自组装薄膜的可控生 长. 制得粒径不同的 $\mathrm{ZnO}$ 自组装薄膜在太阳光照射 下具有良好的光催化降解活性, 并且随着 $\mathrm{ZnO}$ 粒径 的减小, 甲基橙的降解率逐渐增大. $\mathrm{ZnO}$ 自组装薄 膜降解甲基橙的反应遵循一级反应动力学规律, 随 着 $\mathrm{ZnO}$ 粒径的减小, 反应速率逐渐增大.

\section{References}

1 Karunakaran, C.; Dhanalakshmi, R. Radiat. Phys. Chem., 2009, 78: 8

2 Lu, H. M.; Takata, T.; Lee, Y. Chem. Mater., 2004, 16: 846

3 Liao, D. L.; Badour, C. A.; Liao, B. Q. J. Photochem. Photobiol. A, 2008, 194: 11

4 Hu, J. Q.; Bando, Y. Appl. Phys. Lett., 2003, 82: 1401

5 Su, C.; Hong, B. Y.; Tseng, C. M. Catal. Today, 2004, 96: 119

6 Kansal, S. K.; Singh, M.; Sud, D. J. Hazard. Mater., 2008, 153: 412

7 Evgenidou, E.; Konstantinou, I.; Fytianos, K.; Poulios, I.; Albanis, T. Catal. Today, 2007, 124: 156

8 Karunakaran, C.; Dhanalakshmi, R. Sol. Energu. Mater. Sol. Cells, 2008, 92: 1315

9 Zhang, J. H.; Xiao, X.; Nan, J. M. J. Hazard. Mater., 2010, 176:
617

10 Sun, X. M.; Deng, Z. X.; Li, Y. D. Mater. Chem. Phys., 2003, 80: 366

11 Gao, P. X.; Wang, Z. L. J. Am. Chem. Soc., 2003, 125: 11299

12 Zhai, X. H.; Long, H. J.; Dong, J. Z.; Cao, Y. A. Acta Phys. -Chim. Sin., 2010, 26: 663 [翟晓辉, 龙绘锦, 董江舟, 曹亚安. 物理化学学报, 2010, 26: 663]

13 Devi, L. G.; Reddy, K. M. Appl. Surf. Sci., 2010, 256: 3116

14 Nguyen-Phan, T. D.; Pham, V. H.; Cuong, T. V.; Hahn, S. H.; Kim, E. J.; Chung, J. S.; Hur, S. H.; Shin, E. W. Mater. Lett., 2010, 64: 1387

15 Zhang, Y. R.; Wan, J.; Ke, Y. Q. J. Hazard. Mater, 2010, 177: 750

16 Zhu, X. Q.; Zhang, J. L.; Chen, F. Chemosphere, 2010, 78: 1350

17 Vayssieres, L. Adv. Mater., 2003, 15(5): 464

18 Ullah, R.; Dutta, J. J. Hazard. Mater, 2008, 156: 194

19 Xie, J. S.; Wu, Q. S. Mater. Lett., 2010, 64: 389

20 Sobana, N.; Swaminathan, M. Sol. Energ. Mat. Sol. C, 2007, 91 : 727

21 Daneshvar, N.; Aber, S.; Seyed Dorraji, M. S.; Khataee, A. R.; Rasoulifard, M. H. Sep. Purif. Technol., 2007, 58: 91

22 Liu, Z. L.; Deng, J. C.; Deng, J. J.; Li, F. F. Mat. Sci. Eng. B-Solid, 2008, 150: 99

23 Xie, J.; Deng, H.; Xu, Z. Q.; Li, Y.; Huang, J. J. Cryst. Growth, 2006, 292: 227

24 Wang, H.; Yan, K. P.; Xie, J.; Duan, M. Mat. Sci. Semicon. Proc., 2008, 11: 44

25 Yang, H. Q.; Li, L.; Song, Y. Z.; He, P.; Yang, W. Y.; Ma, J. H.; Chen, D. C.; Fang, Y. Sci. China Ser. B-Chem., 2007, 37: 418 [杨合情, 李 丽, 宋玉哲, 贺 萍, 杨文玉, 马军虎, 陈迪春, 房 喻. 中国科学 B: 化学, 2007, 37: 418]

26 Yassitepe, E.; Yatmaz, H. C.; Ozturk, C.; Ozturk, K.; Duran, C. J. Photochem. Photobiol. A, 2008, 198: 1

27 Rao, A. N.; Sivasankar, B.; Sadasivam, V. J. Hazard. Mater., 2009, 166: 1357 